\title{
Functional technology foresight. A novel methodology to identify emerging technologies
}

\author{
Riccardo Apreda $^{1} \cdot$ Andrea Bonaccorsi $^{2} \cdot$ Felice dell’Orletta $^{3} \cdot$ Gualtiero Fantoni $^{1}$ (1)
}

Received: 15 August 2016/Accepted: 21 October 2016/Published online: 22 November 2016

(C) The Author(s) 2016. This article is published with open access at Springerlink.com

\begin{abstract}
The speed and complexity of the technology evolution faced by modern societies need new approaches to the analysis and understanding of the world. Indeed, an exclusive focus on technological goals can miss to recognize all the stakeholders of a technology and address real user needs; moreover, on the one hand low signals are becoming more and more important in fast evolving markets, on the other hand the excess of hype, fashions, or vested interests sometimes deeply alter indicators. However, the so called Big Data promise to be a huge low cost set of valuable information, available and affordable to all (SMEs included). But, analyzing them is not trivial especially if we deal with academic papers and patents. To tackle these issues, the present paper proposes to apply a powerful methodological tool called Functional Analysis to the Technology Foresight process. Actually the rigorous study of the functions, that an artefact should perform to satisfy the user needs, provides a universal and thus unifying point of view, which is able to correlate the user perspective on the product with its technical features. Functional reasoning has been applied to (i) detect possible patterns of development, spotting missing elements and highlighting strengths as well as potential sources of failure; (ii) to enhance traditional bibliometric tools such as the analysis of S-curves and (iii), integrated with a natural language processing analysis toolchain, tailored for patent documents, to identify emerging technologies. The paper describes the functional approach to technology foresight activity, presents
\end{abstract}

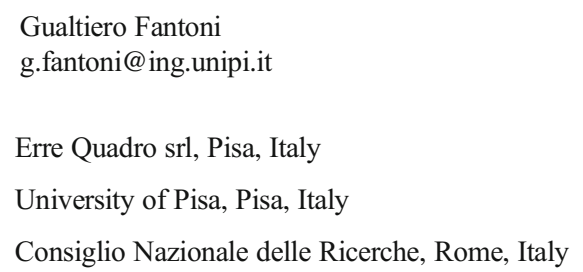

how to integrate it with text mining algorithms and experts' domain knowledge, and finally discusses its benefits in the context of Technology Foresight also from an economic point of view, showing that oresight is affordable also for Small and Medium Enterprises.

Keywords Technology $\cdot$ Foresight $\cdot$ Biomedical $\cdot$ Functional analysis $\cdot$ SMEs $\cdot$ Affordability

\section{Introduction}

Technology Foresight has been used for decades as a tool for decision making in the context of government innovation policies or investment decisions of large companies. By its very nature, it used to be a long and expensive process, largely based on the contributions of senior experts. More recently, participatory Technology Foresight exercises have been undertaken in a variety of countries. While these processes rely somewhat less on the contribution of individual experts, nevertheless they require the mobilization of large resources in order to design and manage meetings, workshops, stakeholder consultations and consensus building sessions. They are also complex to manage and expensive.

With the acceleration of technological change, however, there is a need for Technology Foresight to enter the agenda of SMEs, as well as of public organizations that are less resource-rich than governments, such as municipalities, hospitals, or civic communities. The reason is that technological changes influence to a great extent the life of organizations at any scale. The anticipation of future technologies becomes a crucial component of strategic management, but also, to a certain extent, of operational decision making. To what extent will the sharing economy modify the way in which citizens move, drive a car, rent a house, or visit a tourist place? To what 
extent will neuro-rehabilitation, remote assistance, exoskeletons, telemedicine or telecare influence the way in which elderly people will use health care systems in the next few years? Are drugs to be substituted for by direct electrostimulation of terminals of organs? How does Virtual Reality (VR) and Augmented Reality (AR) will change the way in which companies organize their sales, after sales, logistics, maintenance and support activities?

These questions are no longer the domain of large organizations, either public or private, but become the flesh-andblood of all organizations, of any size.

This however creates a severe challenge: how to ensure that robust, reliable, validated knowledge about the future is made available to less resource-rich organizations. In other words: How to make Technology Foresight less expensive, while keeping its quality high.

A natural candidate to do this job is, needless to say, Big Data. There are currently large expectations about the delivery of customized, timely, reliable information based on the huge computational power of the cloud and the impressive developments of algorithms.

Is Big Data the solution? Yes and no. In this paper we argue that large scale computational tools are not the solution, unless they are applied to a sound, robust, validated domain knowledge platform. We also suggest that this platform already exists, although it has never considered, to the best of our knowledge, as a candidate for Technology Foresight exercises. The platform is offered by the recent developments in Functional Analysis, a multidisciplinary field originated in engineering design theory, but recently enriched by contributions in architectural design, aesthetics, anthropology, and philosophy of technology. Big Data is the solution if it is associated to a robust domain-knowledge platform.

In the paper we present the potential of the combination between new algorithms applied to large scale textual databases and the use of domain knowledge derived from engineering and physics.

\section{Functional technology foresight}

\section{Why functions?}

In order to examine a technology or a product, particularly in the perspective of Technology Foresight, it is important to specify which perspective is adopted.

Before addressing societal needs, let us first study an easier case, i.e. consumer products. Let us consider for example a sailing ship. The marketing staff will work on the values that could motivate the potential users: freedom, adventure, contact with nature and the like, which in turn will imply the need for safety, comfort, speed, acceleration, stability. In the same way the technical personnel will design the hull, the sails etc., using specifications, drawings, computations and choosing materials and components. As another example, consider a washing machine. The marketing staff will work on the values that could motivate the potential users: health, cleanliness, integrity, self-image, social acceptance, which in turn will imply the need for efficient hygienic treatments, reliability, low energy consumption. In the same way the technical personnel will concentrate on the engine, the drum, etc.

These worlds are described using different languages: the language of technical specifications, on one hand, and the language of daily life, on the other hand. Functions constitute precisely the link between the two worlds, the world of technical structures and the one of user needs. Indeed, one definition of function is "the teleological connection between a human's goal and the measurable effects of the artefact" [1]. Another useful definition is "the result of the user's interpretative process about the product's physical behaviours" [2].

Functions can be described in a language which is based on verb + object pairs (with further modal qualifications, if needed), is independent on specific technological solutions, is subject to different levels of abstraction and allow decomposition. Thus in the case of ship, all user needs can be captured and translated into the overall ship functions: to float, to navigate, to (be easy to) control and manoeuvre, all of which can be further decomposed and in turn imply and can be obtained through a series of technical solutions for the boat structure. It is possible to break down secondary functions into more detailed sub functions, and associate them to geometrical or mechanical features of the product so that a deep understanding of each part is gained, as well as of the interaction with the external environment [3].

Recent developments in the literature have offered extremely powerful linguistic resources ("functional bases") that can be used, with some initial training, to develop precise and complete functional descriptions. Functional descriptions can therefore be obtained at the desired level of abstraction, for whatever technology or product (so far, only for entities located above the nanoscale), in a language that is fully consistent with physical descriptions [4-6]. These developments support the promise that Functional Analysis, which has been hitherto confined into a highly specialized and idiosyncratic academic and professional community, is instead largely used to address a variety of issues. This paper builds upon this literature to introduce Functional Analysis into the field of Technology Foresight.

\section{Functional representations}

Rather than a single methodology, Functional Analysis (FA) is a paradigm within the engineering design discipline, which has established a core of fundamental ideas 
over sixty years ago and evolved since then along various directions, both at academic and industrial level. The approach has now entered its maturity phase, even though its applications are still discontinuous. ${ }^{1}$

A detailed illustration of the theoretical aspects of Functional Analysis would be out of the scope of the paper. In the following we will just sketch some of the relevant entities that characterize the functional paradigm and the derived methodologies.

In the classical approach by Pahl and Beitz [7], functions are conceived as "operation on flows" and represented using a pair of words: a verb, describing an action (operation), and a noun, describing the object of that action. The objects are conventionally called flows and classified according to three categories: material, energy and signal. The functional decomposition of a product is therefore given by an ordered list of (verb + noun) pairs.

Clearly, the goodness of such decomposition heavily depends on the goodness of the "vocabulary" used, i.e., on the correct choice of the functional actions and objects. Many research efforts have therefore been focused on the development of standardized, suitable taxonomies and catalogues of all possible functions and flows. It is not an easy task: the functional language should be at the same time general (in order to abstract from specific technical realizations) but complete (in order to encompass all possible solutions); it should be precise (in order to avoid loss of information) but concise (in order not to sink in irrelevant details). Furthermore, the language must be clear, easy to understand also by non experts and easy to use.

As mentioned above, after several decades of research and practice, recent developments in the literature offer restricted vocabularies [9] or large comprehensive vocabularies [5], upon which it is possible to build functional representations that satisfy these requisites. Although there have been critical remarks and extensions [10,11], this line of research is established. As we have argued elsewhere, it is a promising direction for reconceptualising several chapters in the theory of technology and technological change [12].

\footnotetext{
${ }^{1}$ Please note that the meaning of function which is adopted in this paper is related to, but largely different from, the one introduced in the innovation system literature by the "system function" perspective. According to Hekkert [4] and Bergek [7], while there is huge heterogeneity in national and regional innovation systems around the world, there are also some common underlying dimensions, or "functions", which all systems must somewhat perform in order to survive and maintain stability.

These are broadly described as Entrepreneurial Activities, Knowledge Development or learning, Knowledge Diffusion through Networks, Guidance of the Search, Market Formation, Resource Mobilization and Counteracting Resistance to Change (also Support from Advocacy Coalitions). Bergek et al. [8] add Legitimation and Development of positive externalities to the picture. We stick to a definition of functions related to the physical dimension of artefacts (or living systems). However, the link between the two notions should be explored.
}

\section{Ideality and predictivity}

The idea of applying the analysis of functions to Foresight is not new. An influential tradition in engineering literature has claimed that a finite number of evolutionary trends can be identified on the basis of a massive analysis of past technologies, as documented in patents. The TRIZ methodology, developed by Russian scholars and popularized by its founder Altshuller [13], has suggested that it is possible to anticipate technological evolution by applying a small number of such general evolutionary principles to existing technologies [14].

The basic idea is that technical systems evolve following a law of ideality, defined as the maximization of the ratio between useful functions and non-useful (i.e., those not adding any value to the product or even having harmful consequences) ones.

Ideality $=\frac{\sum \text { Useful functions }}{\sum \text { Non-useful functions }}$

Since every function is associated to the satisfaction of a need as well as to a practical effect on the product itself or on the surrounding environment, each useful function will produce benefits (economic benefits, satisfaction of the user and so on) while each negative function will be associated to costs or damages. Therefore it is possible to define ideality also as the ratio between benefits and costs, thus recovering a notion closer to the common perception in the industrial world.

Ideality $=\frac{\sum \text { Benefits }}{\sum \text { Costs }+\sum \text { Damages }}$

The TRIZ literature offers a rich analysis of evolutionary trends aimed at increasing product ideality. If applied to an industry, these trends offer a technological counterpart of traditional economic models of technology evolution, such as Sshaped curves or the Industry Life Cycle model. This allows a combination of various dimensions in a predictive model, as shown in Fig. 1.

According to the S-curve model, the technology is invented at point 0 in time. Initially (phase 1), the development proceeds slowly due to a huge number of implementation problems to be solved and to the lack of profits (actually the technology absorbs money in terms of investment in R\&D). During the growth stage (2) the advantages of the technology become clear and therefore a profitable market develops. By now, many problems have been solved and new investments help performances to improve even more. In the maturity stage (3), profitability and diffusion are at their highest; on the other hand the process of innovation slows down again, proceeding only through minor improvements and approaching the saturation. In the decline stage (4) it is not 
Fig. 1 S-curves for a given technology [13]. 0) is invention, the other four phases of evolution are 1) early stage, 2) growth, 3) maturity and 4) decline
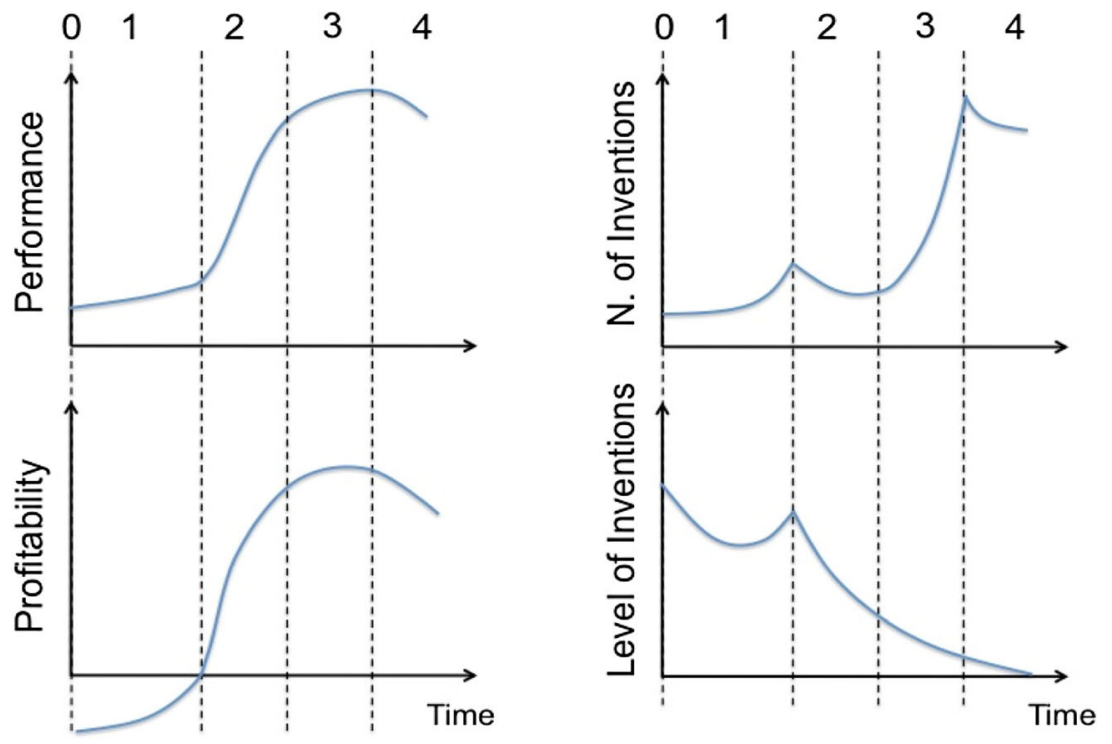

possible to improve the technology further; eventually it will be ousted by a radically new solution.

The crucial prediction of this model is that there is a point in time (point A in Fig. 2) in which it will be more profitable to switch from the old to the new technology, because there will be another point in time (point B) in which the entire market will switch to the new technology as well. Needless to say, the model has limited predictive power on when points A and B will materialize for a given technology. While this model is considered appropriate at theoretical level, its utilization in practice is severely limited by the sheer difficulty in identifying the appropriate level of analysis and predict accurately the switching points.

We suggest that Functional Foresight is an answer to this issue. Focusing on functions, rather than on technology and performance, it complements standard S-curve based analyses and helps to make more abstract and general predictions on the evolution of technology.

This is an important achievement, one that other frameworks find very difficult to reach. In fact, models focusing on the technical aspects only, and TRIZ-based approaches are no different in this respect, applying a restricted set of principles and ignoring the possibility that other forces can be at work.

Furthermore, TRIZ and similar performance-oriented methods require the description of technology to be fully available in engineering language, requiring strong technical background in all participants to sessions and raising the barriers to entry. On the contrary, following recent developments on functional bases and languages, it is now possible to build up complete functional descriptions starting from natural language.

The key to predictivity, as it will be shown below, is the ability of our approach to locate the evolutionary trends and the associated switching points not in the space of technology (or the structure space), but in the abstract space of functions. The condition for this analysis is that the function space is described accurately and completely, something we have demonstrated feasible, at least using a constructive proof approach [15].

\section{Combining functional analysis with bibliometric tools}

Scientific literature is one of the main sources for Foresight exercises. Its use has been profitably coupled with text mining techniques [16]. Kostoff and Schaller [17] review the methods based on co-occurrences and co-citations from bibliometric data. More recently, these techniques have been greatly enhanced by the power of computational linguistics tools applied to web resources (for an interesting example but on a different subject, see [17]).

Patents have also been intensely used as sources of information for Technology Foresight. On one hand, patent bibliometrics, since the pioneering contribution of Narin [18] has been used to identify trends and technology clusters, using both statistical analysis and citation analysis, particularly on non-patent references [19-21]. In this direction, patent analysis is carried out alongside bibliometric analysis [22]. On the other hand, patent network analysis has been recommended as a tool to identify latent structures in emerging technological areas [23-25]. As Verspagen [23] has noted, however, the existing literature lacks a perspective on the "inner dynamics" of the engineering dimension of technological paradigms and the relations with scientific developments [23]. Following the approach of Hummon and Doreain [26], he uses graphtheoretic properties of citation networks to highlight turning points and cumulativeness in engineering developments.

Interestingly, patents have not been utilized to extract one of the most important information content they contain - the functional description of the invention. 


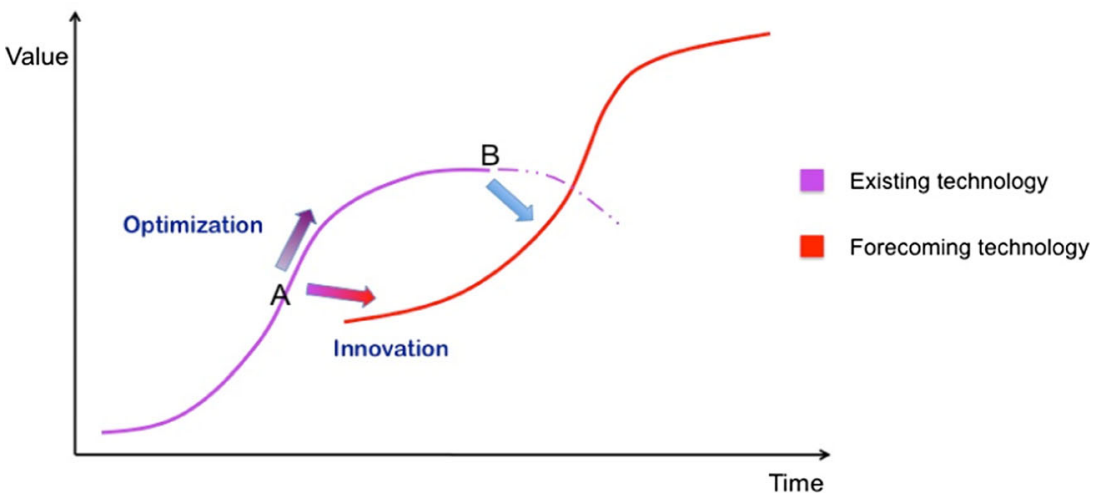

Fig. 2 Types of technology evolution. At a certain point A in time it is possible either to improve existing technologies (optimization), making them more profitable in the short term, or invent a completely new technology (innovation). The new technology will grow along its own

A crucial tool we suggest is Functional Bibliometric Analysis (FBA). It is commonly understood that scientific publications and patents can be scanned in order to identify promising technologies. We apply state-of-the-art semantic software technologies to the tracking of promising technologies not only by using keywords and descriptors, but by searching for meaningful clusters of keywords whose meaning is given by functional lemmas, extracted from full scale functional maps. As it will be shown, this is more powerful than conventional scanning using keywords based on technology.

This should not come as a surprise, given that functions are the most efficient way to capture the key features of human artefacts, but is nevertheless particularly relevant in the context of patent analysis, where sometimes companies hide sensible information behind vague or generic technical descriptions to better protect their competitive advantage.

A risk of traditional queries based on a list of keywords is that the perimeter of the search is fixed by the knowledge of the expert selecting the keywords themselves, and therefore it is difficult to find less known, unexpected or divergent solutions. Moreover, with standard investigations it is not straightforward to disentangle what is really new with respect to the past in the technologies that have been found out.

On the contrary, by contrasting the set of more recent patents with the whole patent corpus, the FBA software tools are able to self-detect, among the unstructured information, those technical solutions that are really new and not just matching a given query criterion.

In addition to finding the positive marks of technologies, the functional semantic extraction is also able to let emerge their potentially harmful or unwanted consequences, another aspect that is not easy to determine otherwise, since not explicit in patents and often overlooked in publications in favour of technical promises and expectations.

The gathered information about advantages and disadvantages can be combined with the study of S-curves and with a
S-curve, eventually becoming more profitable than the old one. Just before that, at point $\mathrm{B}$, the market will switch completely to the new technology

full-scale failure analysis to provide precise indications about the potential of development for a given technology.

\section{The advantages of functional technology foresight}

A first advantage of the use of Functional Analysis in the context of Technology Foresight is the abstract and universal nature of functional descriptions. Let us consider one of the most common objects of daily life, a drinking glass or cup. It has two main functions, the first being of course to contain liquids. Such function is however shared with other containers such as bottles, barrels and the like; what distinguishes a glass from bigger recipients is the ability to set the amount of contained liquid to a reasonable quantity (usually one or two mouthfuls), in order to be held and brought to mouth without effort.

The so-called secondary or auxiliary functions will also tell what is necessary to make a good glass or cup. These functions are not indispensable as such, but their implementation would make the difference between a successful product and an unsuccessful one. It does not really matter whether the cup is made of glass, plastic or wood, neither does its colour or shape. What matters for a good glass is to be stable while standing on a table and while pouring liquid, to be easy to grasp, to have a good match with human hands and lips, and to properly guide the liquid into the mouth when drinking. The functional requirements will then suggest the right material or the right shape to use, and rule out the wrong ones. It is not a coincidence for example that almost all cups are roundly shaped: a cup with a square section is not practical to grasp, it does not match the lips' shape and water tends to spill out at the edges. In other words, functions capture the user needs (to drink without nuisances) and translate them into the proper technical specifications.

Let us now remark the generalizing power of such representation. Any object that fulfils the two main functions is, almost by definition, a drinking tool. Two abstract functions describe the essence of a glass and six (considering the four secondary functions) the essence of a good, successful glass. 
Even distinctive aspects of specific glasses can be described using few additional functions (e.g., wine glasses use their shape to keep the aroma and direct it to the nose, and to isolate the liquid from the body's heat). Due to its power of abstraction, Functional Analysis is the appropriate methodology to deal with almost all dimensions of technological innovation. As an example, the study of functions proved to be extremely useful in generating new ideas and concepts and in guiding the innovation process.

A second advantage of the approach is that it provides a unified point of view for describing human artefacts. This is done by creating a shared language between technologists (both from academia and from enterprises) and stakeholders (users, commercial partners, policy makers and so on), two communities which otherwise would hardly understand each other. Engineers and scientists would pursue better performances and talk about technical details, while stakeholders would focus on satisfying needs or solving problems, but would not be able to define them rigorously enough, to examine the implications of different technological options, or to propose implementations. This is an enormous advantage in the context of societal challenges, for which the gap in understanding each other between society and technology may be large.

Functional Analysis offers a well-structured bridging language that can translate the terminology and expressions of one world into the ones used in the other. Indeed, our experience is that after having built the proper functional representation for a technology, the dialogue between the two parties becomes much more concrete, fluid and creative. Participants do not need to be educated in engineering to enter into discussions of technology, but after an initial (relatively short) training they can start discussing options at the appropriate level of abstractions.

Third, a distinctive property of functional language is to facilitate the identification of technology trends, or the long-term directions of technology in specific fields, and of technology options, or the bifurcations that may take place along longterm directions. With functional forecasting, it is possible to apply general trends (such as those identified in the TRIZ literature $[13,14]$ to a specific technology, and to study functional variants.

By focusing on abstract functions, rather than on specific solutions, such approach allows a systematic comparison between current technology and the frontier, as will be discussed below. In the same way, it allows a proper comparison of products, even in the case of products that are very far from a technical point of view. It goes without saying that the possibility of such comparison is very useful in Technology Foresight, where one has to assess the potential of competing technologies, and the focus on other dimensions such as product performance could be misleading and potentially dangerous, as extensively discussed in [23]. In doing so, a functional view greatly increases the flexibility of decision makers, by clarifying the implications of all technology options and limiting the commitment to given solutions.
In addition, a functional view is very useful to identify what is missing in the technological perspective. As a large literature in STS has shown very often, what is missing, is a deep consideration of changes in work practices, skill deployment, or complementary skills, induced by technological change. Oudshoorn [27] has recently shown how the introduction of telecare in health systems has failed to take into proper account the professional skills of doctors and nurses for the new delivery system. It is our contention that a higher level, functional view would have helped to identify missing elements of the technology, as the case study below will show in detail.

Finally, functions naturally embed the information about user needs, along with the information about physical realization, and are therefore more suitable to point out potential failures of developing technologies in meeting the market or societal expectations [28]. In the same way the study of functions allows to highlight the presence of hidden negative functions that can undermine the potential of certain otherwise successful technologies (see [29] for a comprehensive application of failure analysis techniques to Foresight and for a case study in the textile industry). The possibility to spot hidden failures in advance is a powerful antidote to contrast the effects of hype, excessive expectations and even of vested interests in the decision making process.

\section{A case study in the medical device industry}

\section{Introduction}

The functional approach and the tools described in the previous section have been applied to the analysis of the medical device industry, a rapidly growing sector characterized by high technology content, often at the frontier of scientific research, and therefore rich in promises as well as in uncertainties about future developments.

The study was performed as part of an innovative Technology Foresight exercise commissioned by the Regional Government in Tuscany, Italy, and carried out in 2010-2011, publicly presented in 2011 and published in 2013 [30]. The main goals of the investigation were to evaluate the emerging technologies, to identify the most promising products, and to anticipate the scientific and societal inputs that will influence the evolution of the medical device industry. The information thus gathered will help the Regional government to focus and prioritize its investments into R\&D support.

The study was performed combining three different approaches, so that the results could be cross-checked. First, traditional methods of analysis have been used, such as expert panels [31], consensus building, SWOT tables and roadmaps, mainly to circumscribe and define with precision the objects of interest and the boundaries of the investigation. Second, Functional Analysis has been used to re-structure the gathered information, 
producing full-scale functional maps of all involved technologies. Using the functional maps, a number of technology trends have been identified, translated into roadmaps, and discussed extensively with stakeholders. Finally, Functional Bibliometric Analysis (FBA) was used to identify the relevant patent and publications literature on future trends.

\section{From technology-based segmentation to functional segmentation}

The first step of the analysis consisted in the gathering of information from human experts and by processing industry publications, newsletters, websites and the like. This activity led to the selection of a list of products (or families of products), considered most innovative or at least very promising in the medical device industry. These are displayed in Table 1.

On each of them a quite conventional technological analysis has been carried out, following a structured questionnaire. A panel of five senior experts (from academia, industry and consulting) was hired to support and guide the analysis, while the results were discussed with a large group of stakeholders, including approximately forty among managers, start up entrepreneurs, patent consultants, trade association representatives, public health care officials, and hospital managers.

Starting from this material, technologies have been decomposed following functional criteria and these have been used to produce a functional segmentation (that is a structured reorganization following functional principles). Combining the technological approach and the functional decomposition/ segmentation resulted in a large full scale functional map, initially realised on paper, eventually crafted as a digital product and shared among experts and stakeholders (Fig. 3).

The map clusters technologies around broad functions, and offers for each technology a descriptive label based on technology/product and market/application. The labelling is important in order to keep the abstract functional description within a concrete context. At the same time each technology is placed within a broader and more abstract context, described by functional verbs.

\section{Identifying technology trends in the functional map}

A full-scale functional map is a powerful cognitive support for a number of creative activities.
Table 1 List of medical device technologies examined during the Foresight exercise

\begin{tabular}{|c|c|}
\hline HIFU & $\begin{array}{l}\text { High-Intensity Focused Ultrasound - a medical procedure that uses focused } \\
\text { sonic energy to heat and destroy tumoral tissues. }\end{array}$ \\
\hline NOTES & $\begin{array}{l}\text { Natural Orifice Transluminal Endoscopic Surgery - a minimally invasive } \\
\text { technique that uses human body's natural orifices to introduce surgical } \\
\text { instruments and reach internal organs, with no external incisions or scars. }\end{array}$ \\
\hline Harmonic ${ }^{\mathrm{TM}}$ Scalpel & $\begin{array}{l}\text { A surgical scalpel that uses high-frequency vibration to cut through tissues; } \\
\text { at the same time it achieves haemostasis, sealing blood vessel by coagulation. }\end{array}$ \\
\hline TissueLink $^{\mathrm{TM}}$ & A surgical scalpel that uses radiofrequencies to cut and achieve haemostasis. \\
\hline Coblation ${ }^{\circledR}$ & A device that induces ionization to dissolve target tissues at low temperatures. \\
\hline Cyberknife ${ }^{\circledR}$ & A robotic system for radiosurgery (treatment of tumors using X-ray radiation). \\
\hline Echomyograph & $\begin{array}{l}\text { A device integrating an ultrasound scanner and an electromyograph to achieve } \\
\text { the simultaneous evaluation of results. }\end{array}$ \\
\hline Advanced urodynamics & Combination of ultrasound scans with traditional urodynamic testing. \\
\hline RFID applications & $\begin{array}{l}\text { Radio Frequency Identity Devices allow automatic objects' identification; many } \\
\text { uses are possible, from tracking drugs to monitoring patients conditions. }\end{array}$ \\
\hline Neuro-rehabilitation & $\begin{array}{l}\text { Use of mechatronic systems for post-trauma motor rehabilitation; it allows new } \\
\text { protocols, telecare solutions, and treatment of larger numbers of patients. }\end{array}$ \\
\hline Laser regeneration & Laser radiation is used to induce tissue regeneration (e.g. in skin treatments). \\
\hline Dedicated MRI & $\begin{array}{l}\text { Smaller, transportable MRI devices able to can scan single body parts; they can } \\
\text { be used for a variety of exams, e.g. to study bone pathologies. }\end{array}$ \\
\hline Micro CAT & Smaller, transportable CT devices to scan single body parts or small animals. \\
\hline PACS & $\begin{array}{l}\text { Picture Archiving and Communication System - medical imaging databases for } \\
\text { improved management, sharing and statistical elaboration of data. }\end{array}$ \\
\hline Wearable sensors & Clothes-embedded sensors monitoring medical parameters for early diagnosis. \\
\hline Colonoscopy pills & Pills containing video cameras can substitute standard, invasive colonoscopy. \\
\hline Induced ultrasounds & Laser-induced ultrasounds can be used to achieve non-invasive virtual biopsies \\
\hline CMUT systems & $\begin{array}{l}\text { Capacitive Micromachined Ultrasonic Transducer - innovative transducers } \\
\text { allowing smaller dimensions, larger bandwidth and higher resolution. }\end{array}$ \\
\hline Electric pain therapy & Electrical stimulation of tactile nerves may help reducing pain sensations. \\
\hline
\end{tabular}




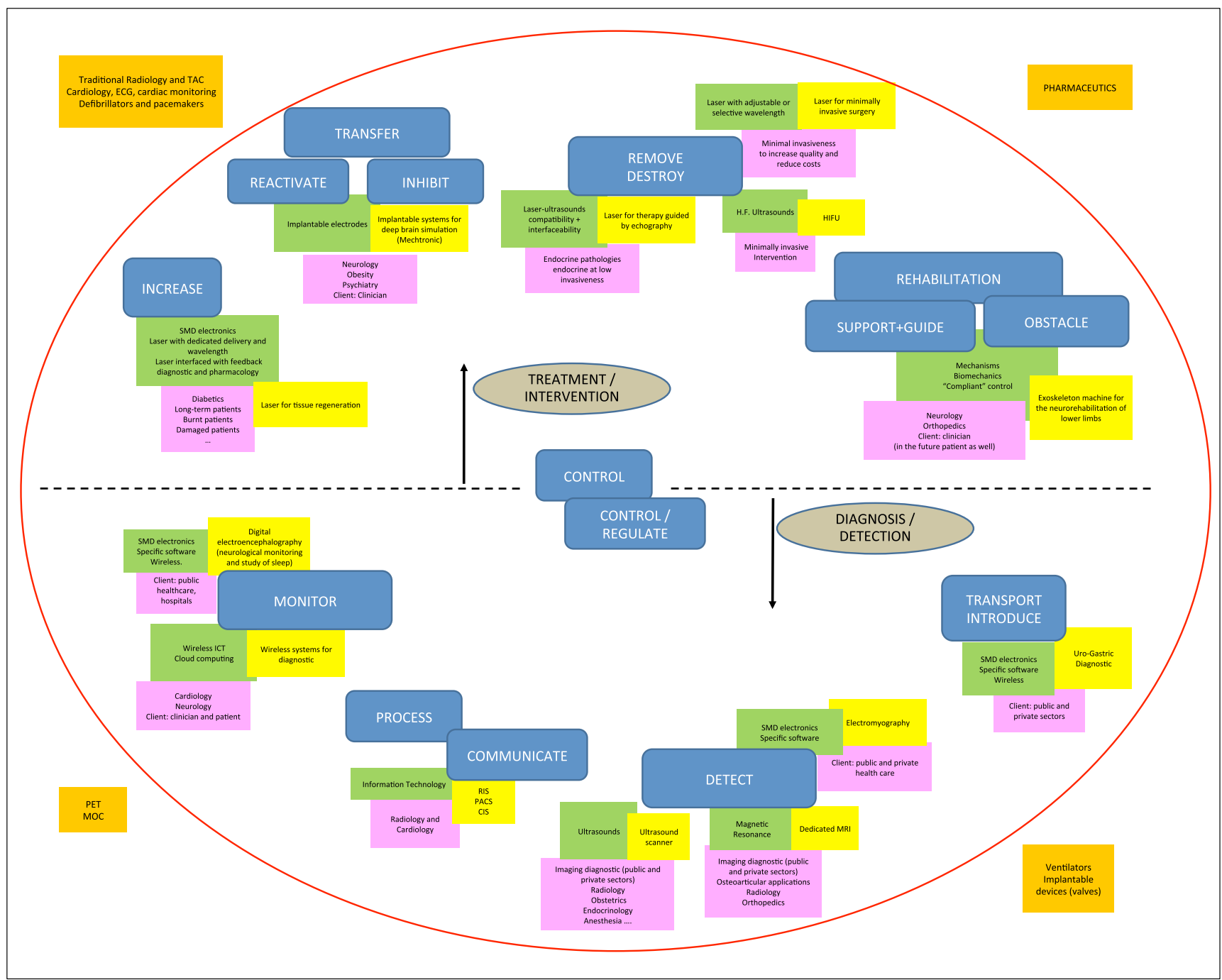

Fig. 3 Functional segmentation of a set of medical devices. Products (yellow boxes), corresponding technologies (green boxes) and markets/ applications (violet) are grouped around functions (blue boxes) in the two

In the field of product innovation we experimented with using creative problem solving heuristics (such as analogy, crossover, recombination, and the like) directly within the functional map in order to identify new product ideas (see for example [32, 33]).

Here, in the context of foresight we adopted a structured approach: we projected all dimensions of functions in the future, by changing systematically the objects or the modalities involved in the functional description. This resulted in a large number of potential evolutionary trends, which we submitted to thorough technical evaluation. Finally, we discussed these trends with stakeholders, with surprisingly good results in terms of clarity of perspective and precision of suggestions.

Table 2 offers a list of technology trends that are considered relevant from both user and producer perspectives.

For example, consider the trend towards integration between diagnostic and therapeutic functions in the same device. macro-areas of treatment and diagnostic. The topics in the orange boxes at the corners indicate those biomedical fields that were willingly considered outside the perimeter of the investigation

The same pattern emerges clearly (even visually) when comparing two temporally shifted functional segmentations such as that in Fig. 3, noticing the increased positioning of new devices across the two areas.

It was possible to project this trend into the future by examining the respective functions, decomposing them into elementary sub-functions, specifying the nature of state variables changed by the action, and measuring the physical variables involved. Thus for example if the therapeutic function involves the activation of a targeted laser ray in a small region of a tissue, what was investigated was the compatibility of this function (in terms of precision, guidance, tolerance, thermal impact, biochemical reactions) with the diagnostic function (in terms of bandwidth of the signal, noise, quality of image or sound).

To the surprise of most actors involved, including senior experts, this method in a few cases delivered 
Table 2 Selected technological trends found in the medical device industry. Rather than being uncorrelated, various trends actually strengthen each other, e.g. miniaturization supports both the integration in super-systems and targeted action

- Minimally invasive technology (reduction in manual access, also in diagnosis)

- Integration between diagnostic and therapeutic functions in the same medical device

- Trend towards super-systems (e.g. echomyography = ultrasound scan + electromyography)

- Crossover of solutions to other fields (e.g. cardioneurology)

- Targeted action (e.g. substance delivery)

- Elimination of human involvement (e.g. rehabilitative robotics)

- Increase in the degree of segmentation of the active elements, or trend Mono-Bi-Poly (e.g. tools for the removal of organic materials)

- Miniaturization of devices

- Integration with genetic screening

- Increasing importance of software for support to early diagnosis, image processing and interpretation, simulation of therapies, definition of therapeutic protocols

solutions that were not yet clear to them, but were at a very preliminary stage in exploratory research. In other words, it was possible to foresee the emergence of certain technical solutions just as the natural following steps of some existing evolutionary trends, and a later investigation found out that such solutions were actually under study by research groups around the world.

Needless to say, technical solutions that deliver the next step in a trend in act in a certain sector have a greater chance of success and greater potential, while products that innovate only minor aspects but do not improve over the present evolutionary stage are far less competitive.

Another important implication of the methodology was that technological views of actors involved, particularly those from industry, were challenged.

Therefore technology trends were not obtained by extrapolating current activities in R\&D, but combining expert and stakeholder view with insights generated through an abstract representation, which opened options and challenged established views.

\section{Involving stakeholders}

Does Functional Foresight lead to technological determinism? Definitely not. Functions are described in physical terms, but reflect the teleological relation between the behaviour of an artefact and the needs of a user. In our own definition, a function is "the result of the user's interpretative process about the product's physical behaviours" [2]. The fact that we insist on the use of physical variables [5] is linked to the need for a complete and rigorous description, which is needed for a scientific foundation of Functional Analysis. But functions do not exist outside the user perspective, at least a potential one. Functions are inherently normative [10,12], implying a perspective of relation between a behaviour describable in physical terms and a user.

Based on these firm methodological grounds, we submitted the functional maps and the trends to several stakeholders, both in group meetings and personal interviews. The user perspective added a number of interesting issues, leading to clarification of potential problems in technological trends, sometimes pointing to possible bifurcations.

For example, the trend towards integration between diagnostic and therapeutic functions in the same medical device was discussed in terms of the required changes in the skills of doctors, with deep implications in terms of patients' attitudes (trust), administrative procedures, doctors' status, liability and insurance issues, and the like. From the user perspective, there will be great advantages (in the same session the disease is identified and cured), but also sources of anxiety (there is no longer a time interval between diagnosis and therapy, which could be used by the patient and her family to make an informed decision, or to take a second opinion). The identification of these problems in functional terms, as opposed to extrapolation of existing devices (with associated conflict of interests of industry involved) helped to frame the discussion in a transparent way, reducing the usual suspect of vested interests from the industry side, or manipulation from hospitals.

As another example, within the broad trend of elimination of human involvement, we discussed the concept of rehabilitative robotics. This concept, borrowed from robotics and mechatronics, implies the substitution of the hands of the physiotherapist for automatic operations actuated by robots (usually exoskeletons) and controlled by sophisticated software that controls for physiological parameters, the neurological activity and the muscular reaction of patients, and other factors. Here there are deep social implications. On the one hand, patients will be asked to trust machines instead of humans. The extent to which this delegation of responsibility is perceived with anxiety has been discussed extensively. On the other hand, professional skills of physiotherapists will be challenged. In principle, there might be a new division of labour between humans and devices, with the former concentrating on the high end, sophisticated operations.

Again, working on functional descriptions helped to discuss these issues more thoroughly and dispassionately. These insights were also challenged to regional authorities for health care (a regional responsibility in the Italian institutional framework), which included them into technology assessment activities and into the drafting of health plans.

On the basis of these analyses and discussions we ended up, for a subset of technologies, with a full fledged roadmap. The roadmap includes not only the traditional 
dimensions of technology, product and market/application, as discussed in the literature (e.g. $[17,24])$. It includes explicitly a user dimension (needs) and an environmental dimension (events), aimed at examining broad societal changes, or regulatory activities. An example of roadmap is given in Fig. 4.

Thus the Functional Foresight methodology is not only more powerful and flexible from the technology side, but also more productive on the stakeholder side. It is a candidate for addressing societal challenges by making the technologysociety conversation more productive.

\section{Cross-checking}

Although the functional investigation had already delivered striking insights that gathered the consensus of both experts and stakeholders, the Foresight exercise included also a further cross-check of the results through other methodologies, such as the analysis of S-curves.

The same additional data were also used to integrate the results coming from the Functional Bibliometric Analysis that will be presented in the next subsection.
Of the four parameters that can be used to construct Scurves (see "Big data meets domain knowledge: the functional bibliometric analysis" section), we decided to study the number of inventions over time, since the other three parameters (profitability, performance and level of invention) are not as easy to determine or assess. On the contrary the number of inventions is directly related to the number of patents and scientific papers, which are both already gathered in fully accessible and exhaustive databases. In particular, the study of patents has the highest likelihood of providing complete and accurate results.

Two cases of S-curves for technologies at different maturity stages are shown in Fig. 5.

Apart from the indications that such diagrams give on their own, their relevance increase when put in correlation with other sources of information. It is interesting to note for example that diagrams such as the case a) in Fig. 5, representing emerging technologies with great potential for innovation and profitability, are more often associated to areas of the functional segmentation (see Fig. 3) where fewer solutions are present to satisfy the user's needs expressed by each function. Here in particular we are referring to the painless colonoscopy technologies where

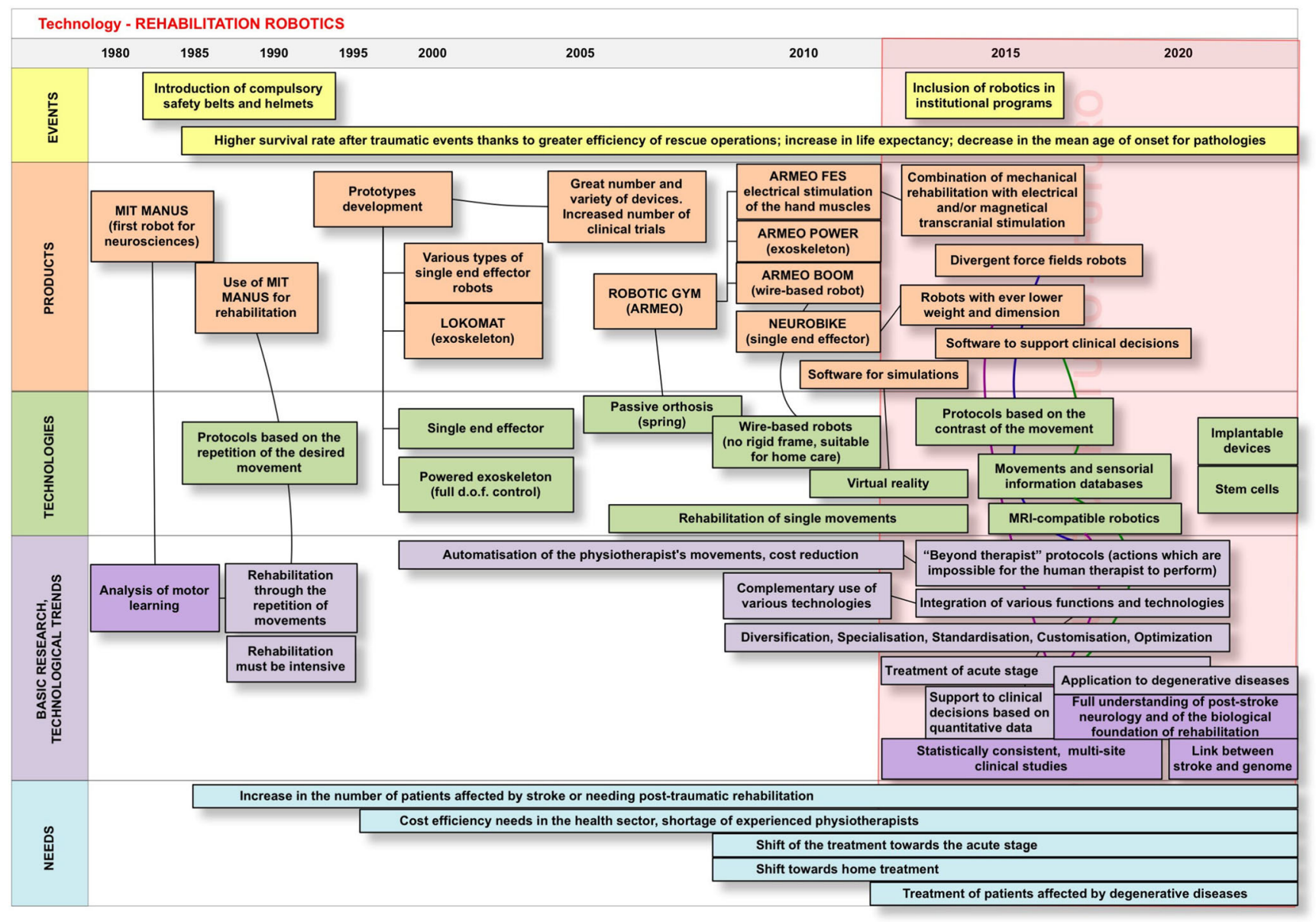

Fig. 4 A combined technology-user roadmap for rehabilitative robotics 


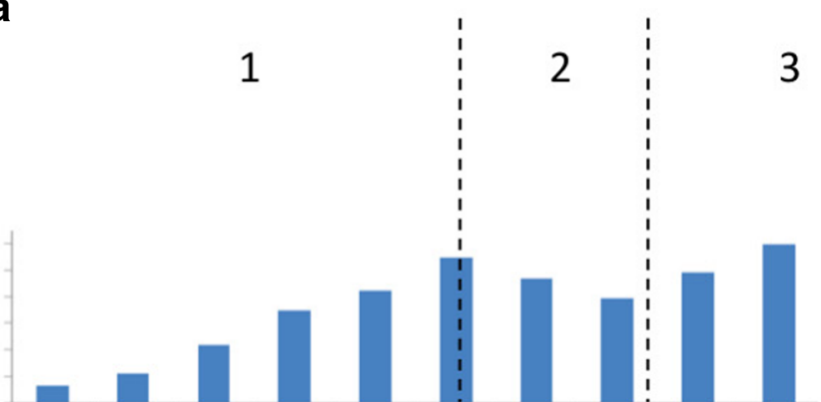

Fig. 5 Examples of S-curves constructed from bibliometric data for two different technologies. Y-axis indicates the number of invention (see Fig. 1) as determined from the number of patents on the topic. The histograms span the same ten years reference period, but have been

we located both soft robots (worm-like endoscopy by EraEndoscopy ${ }^{2}$ ) and Capsules for endoscopy (Apreda et al. 2016 Technology Foresight based on functional analysis, submitted), namely eatable capsules provided with micro video devices, aimed at making pictures of the organs). In 2010, such technology (both capsules and soft robots) was in the early stage but very promising with respect to the standard rigid colonoscopy air inflation and rigid tubing negatively affected the patient during the analysis.

As an additional example, we enquired experts and stakeholders about technologies associated with diagrams such as the case b in Fig. 5), representing apparently declining solutions. In the case of Fig. 5b we are referring to HIFU technology, namely High intensity focused ultrasound. Such technology focuses ultrasound waves in a targeted way against tumoral areas but not damaging surrounding organs. ${ }^{3}$ In 2010 we found evidences that the HIFU was associated with a series of drawbacks. Therefore we consulted external medical doctors who pointed out the existence of collateral damages or other factors limiting the further success of the technology. Nowadays HIFU is limited to a very small subset of tumours and medical applications than the high number claimed in 2010 (please see the evolution history in Wikipedia page from 2010 to now).

Nowadays, when we recently contacted again the experts (June 2016), they explained how very recent research solutions solved some of the problems that blocked the HIFU development, thus probably shifting the technology over a new S-curve.

Finally, many of the outcomes of this stage of the investigation were confirmed by the subsequent Functional Bibliometric Analysis, which extracted from the literature the positive and negative keywords associated to the various technologies. For a more detailed description of the abovementioned cases please refer to (Apreda et al. 2016 Technology Foresight based on functional analysis, submitted).

\footnotetext{
2 http://www.endotics.com/,

${ }^{3}$ https://en.wikipedia.org/wiki/High-intensity_focused_ultrasound
}

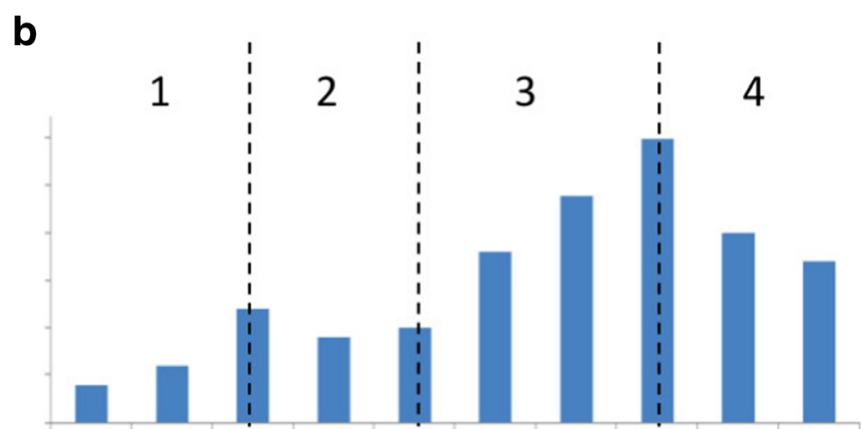

normalized for a clearer comparison. Technology a) is just entering its maturity phase at the end of the survey period, while technology b) is likely entering the decline phase (see again Fig. 1 for comparison)

\section{Big data meets domain knowledge: the functional bibliometric analysis}

The functional methodology also helped to develop a new bibliometric tool, aimed at identifying emerging technologies. The issue here is to combine powerful semantic technologies for automatic processing of data with interpretative power.

The methodology we developed unfolded as follows.

Four groups of patents were identified as relevant for our analysis (Group A61B 8/00: Ultrasound diagnostics; Subgroup A61B 18/18: Laser therapy; Group A61N7/00: Ultrasound therapy; Subgroup A61B17/02: Devices to keep wounds open). A total of 6107 patents were extracted from databases for the period 2000-2010 with their full text.

We then used last generation semantic technologies at that time $^{4}$ to carry out automatic Domain-Specific Entity Extraction.

The term extraction method we followed combined NLP techniques, linguistic and statistical filters. The input text was firstly tokenized, morphologically analysed (i.e. PoS-tagged) and lemmatized passing through a pipeline of state-of-the-art NLP tools for the analysis of texts. The extraction of domain-specific terms or entities follows the methodology described in [38]. The automatically POS-tagged and lemmatized text was searched for candidate domain-specific terms, expressed by either single nominal terms or complex nominal structures with

\footnotetext{
${ }^{4}$ This contrastive method is currently implemented in the T2K tool [34] and it is tested and specialized in the framework of different projects aimed at extracting and organizing knowledge from different Italian and English domain-specific corpora. It has been used for "Social sensing for breaking news" where it supported journalists in analysing Social Media to discover news; Legal Text Mining: building semantic networks to support advanced queries in legal textual corpora (JURNET)*, intelligent Semantic Liquid eBook, aimed at augmenting digital educational publishing with NLP-based functionalities; moreover it has been used also in scientific works: The contrastive analysis performed by T2K was used by Ferrari et al. [35] to extract domain-specific entities from brochures; by Fantoni et al.[36] for detecting and extracting information about the functions, the physical behaviour and the states of the system from patents; by [37] to measure and improve the completeness of the requirements by extracting relevant terms and the relevant relationships among terms from the input specification documents.
} 
modifiers (typically, adjectival and prepositional modifiers), where the latter were retrieved on the basis of a set of POS patterns encoding morpho-syntactic templates for multi-word terms. According, T2K extracts domainspecific entities typically expressed through nominal (either single or complex) terms. The domain relevance of terms was weighted on the basis of a combination of "termhood" measures, assessing the likelihood of being a valid technical term and contrastive methods. In particular, the terms were weighted with the C-NC value [39], a well-known method for terminology extraction. The ranking of identified terms was then revised on the basis of a contrastive score calculated for the same terms with respect to corpora testifying general language usage. This ranking reflects the domain relevance of terms which is computed on the basis of their inter-domain distribution. It should be noted that the contrastive function is only applied to a top list of these pre-selected terms weighted with the $\mathrm{C}-\mathrm{NC}$ value, which was customised through empirically defined thresholds. This procedure allows focusing, firstly, on the retrieval of valid technical terms, thanks to the statistical filters, and secondly on domain pertinence, in two distinct but consequent moments. The top-list of single and multi-word terms were contrasted firstly against the term list extracted from an opendomain corpus and secondly against a top list of terms acquired from a corpus at the level of the different regulated-domain. In both contrastive phases, the contrastive function (CSmw) newly introduced in [38] was used. The $\mathrm{CSmw}$ score is oriented to prune common words from the list of domain-relevant terms.

Therefore, in the present case text of patents was parsed, tokenized, examined grammatically and lemmatized. After this initial processing a number of single and multiple keywords have been extracted and annotated starting from the functional maps obtained in the previous stages of the research. The conventional keyword search, however, fails to identify the latest trends. A massive interpretation work is needed to reconstruct the inherent technological evolution. Our aim was to develop a tool for fast identification of hot topics. Hence we adopted the contrastive procedure described above by firstly "subtracting" generic terms from extracted terms, and subsequently subtracting keywords obtained in the period 2004-2005 from the sample of keywords obtained in 2009-2010. This double "subtraction" procedure helped to isolate innovative terms appearing for the first time in recent years.

It resulted in a large repository of keywords, of various generality. The next step is the most demanding-interpretation. How could these documents be interpreted without reading all of them? Here the functional methodology comes into play. We included in the keyword search all functional information identified in the previous steps. We then carried out cooccurrence analysis in order to obtain clusters of cooccurring keywords expectedly associated to full meaning.

The approach proved effective. All clusters of keywords were associated to functional verbs that illuminated the semantic content of the documents. People with a general training in engineering could interpret correctly the documents, without the need for domain experts.

Table 3 offers an example of retrieved clusters of keywords. It is an extract of only three clusters in the ultrasound domain, out of around one hundred for all four IPC classes. As it is clear, the functional verbs included in the cluster (e.g. measure, check, abrade) were instrumental in understanding the relations between the objects described by substantive keywords.

Again, to the surprise of experts, the methodology was able to find out emerging technologies that even they were not aware of, or that simply forgot to mention during the interviews.

The first row of Table 3 is a striking example of this case, as well as a very clear example of the results potentially obtainable. The contrast procedure clearly singled out the multiword expression "bone age" as a very recent and meaningful entry in the patent database. At first it was not obvious which technological innovation was involved. It was however enough to read the various words co-occurring with "bone age" ordered according to their syntactic/semantic role in a possible sentence, to get: "bone age" [is related to the] "assessment/ measure" [of the] "skeletal/metaphysis/wrist" "growth" [in] "children or premature babies", [or also] "osteoporosis" [in] "adult", [using] "ultrasound scan". Indeed the experts confirmed that bone age is the degree of maturation of a child's bones, and it is particularly relevant to assess it for premature babies, looking at the wrist's bones; traditional bone scans use X-ray that may be harmful to young children, and one of the most promising research direction (with only one device already on the market) is the use of the much safer ultrasounds instead of X-ray. The second row of Table 3 provides a similar example for a cardiovascular application.

Within the cluster of correlated keywords, it is also possible to find indications about positive or negative features of the technology. For example, in the third row of Table 3 an application of ultrasounds in the therapy of various pathologies has at least four outcomes of medical relevance; one of them is an unwanted collateral damage to a particular type of cells, while another is an unexpected positive feature, an activation of cells that facilitate drug delivery.

The most interesting implication is that the software is able to extract from the existing literature even those negative aspects that are sometimes hidden by official presentations or not mentioned by stakeholders because of vested interests.

The authors are currently developing a more sophisticated version of the bibliometric tool, able for example to determine 
Table 3 Examples of emerging technologies in the sector of ultrasound devices. The first column contains the keywords extracted with the contrast procedure explained in the text, plus the ultrasound filter; the second column lists the other relevant words that recur associated with them, reorganized according to syntactic or semantic similarity in order to facilitate interpretation; finally the third column provide the interpreted description of the technology

\begin{tabular}{|c|c|c|}
\hline Main keywords & Co-occurrences & Interpretation \\
\hline Bone age, ossification, ultrasounds. & $\begin{array}{l}\text { Assessment, measure; } \\
\text { structure, year, growth, velocity, stature; } \\
\text { skeletal, metaphysis, wrist, cartilage; } \\
\text { child, baby, birth, premature; } \\
\text { adult, osteoporosis. }\end{array}$ & $\begin{array}{l}\text { Technique to evaluate skeletal growth in small children } \\
\text { or elders, using ultrasound instead of X-rays. }\end{array}$ \\
\hline Great thickness difference, ultrasounds. & $\begin{array}{l}\text { Diagnostic, measure, check; } \\
\text { elastic, properties, strain, pressure, value, } \\
\quad \text { variation, minimum, maximum; } \\
\text { tissue, vascular, wall, cycle, cardiac; } \\
\text { accuracy. }\end{array}$ & $\begin{array}{l}\text { Technique to evaluate change in thickness and elastic } \\
\text { response to strain of the cardiovascular apparatus } \\
\text { using ultrasounds }\end{array}$ \\
\hline Endothelial cell, ultrasounds. & $\begin{array}{l}\text { Vessel, blood, clot, thrombus, thrombin, } \\
\text { thrombogenic, fibrin, platelets, flow; } \\
\text { damage, hifu; } \\
\text { coagulation, cavitation; } \\
\text { occlusion, catheter, abrade; } \\
\text { activation. }\end{array}$ & $\begin{array}{l}\text { 1. Treatment of thrombus } \\
\text { 2. Damages to endothelial cells (collateral effect of treatment) } \\
\text { 3. Coagulation (haemostatic effect) } \\
\text { 4. Activation of cells to facilitate drug delivery }\end{array}$ \\
\hline
\end{tabular}

the relevant patent classes and documents automatically, to find even concealed information or facilitate crossover from different technological fields.

\section{Will Technology Foresight ever become an affordable luxury?}

One well known trend in consumer products is called "affordable luxury": items such as clothes, furniture or jewels are sold at affordable prices as a second line with respect to fashion-driven, original, designer-identified products.

So far, Technology Foresight has been an expensive luxury for most organizations. Does Big Data hold the promise to reduce the price at which high quality Foresight exercises can be purchased?

We offer here some back-of-the-envelope calculations about the case study discussed above, as a first approximation of the problem. More systematic studies might be done to address this issue.

The Foresight study performed on behalf of the Tuscany Region involved 5 experts, 10 analysts (1 physicist, 2 mechanical engineers, 2 management engineers, 2 economists, 3 software developers), plus 2 senior and 2 junior market analysts.
The experts have been involved for 5 days in meetings, brainstorming, etc. and several skype calls each. The technology analysts participated to the meetings to provide data and insights during the meetings and spent around 20 days of backoffice activity each, the marketing analysts spent about 2 months in preparing the market analysis. The total amount invested by the Tuscany region was in the order of 75-100€.

Since the interesting results and visibility of the study, the research group and a spin-off company of the University of Pisa (Erre Quadro SRL) invested in automatizing the data gathering and analysis phases of the Foresight process. The result is an optimized toolchain and workflow that can be executed (the number of experts depends on the field to analyze) by half of the analysts (both technological and marketing) and a considerable less time to be performed.

From an economic point of view, the total cost for a new foresight is reduced by a factor of 3-4.

Table 4 shows how an optimized workflow with a series of automatized steps can dramatically reduce the necessary effort and time consumption. The four cases in Table 4 have been done in the last years and have been performed for SMEs, thus demonstrating that foresight studies have become interesting and affordable also for them (which are more and more exposed to Foresight methodologies [40]).
Table 4 Estimate of effort of external experts and analysts in a selection of foresight exercises

\begin{tabular}{lllll}
\hline Topic & Expert involvement & Analysts & Elapsed time & Year \\
\hline Energy from sea waves & 1 external expert for 3 half days & 1,5 & 1,5 month & 2012 \\
Batteries & 1 external expert for 2 days & 2 & 1 month & 2015 \\
Electrochemistry & Customer input & 1 & 15 days & 2016 \\
Packaging industry & Customer input & 1 & 1 month & 2016 \\
\hline
\end{tabular}




\section{Conclusions}

The paper has shown how a relatively mature engineering approach, Functional Analysis, can be usefully applied to Technology Foresight issues. Based on recent developments in the creation of functional languages and repositories, the process of construction of functional representations is smooth and relatively easy to learn.

The paper has also shown the potential relevance of nextgeneration bibliometric tools, able to process huge amount of data (technical texts) and based on semantic as well as functional inputs. The results are easy to be read and interpreted by human beings, experts or not.

These developments hold a promise for making Technology Foresight accessible to small organizations, including SMEs and public administrations.

The approach has been presented taking into account a real case performed for the Tuscany region in the biomedical field where a large number of patents, papers and technical publications exist. The paper presents the results of a Foresight performed in 2010-2011 and the provided examples give evidence that the main previsions of technology evolution, flattening or decrease are confirmed.

In cases where the information are hidden or less codified than in the medical or biomedical field, the functional approach can be used as well, but the exploitation of the Big Data with text mining tools has to be substituted with other techniques as shown in [29].

Acknowledgments The financial support of Regione Toscana Project LILIT: I Living Labs per l'Industria Toscana (PAR FAS REGIONE TOSCANA Linea di Azione 1.1.a.3) is kindly acknowledged.

Mr Francesco Galli and Donata Gabelloni, PhD, are kindly acknowledged for their support during the bibliometric analysis, Mr. Gabriele Antonelli and Luca Barsotti for the market analysis and, last, Prof. Gaetano Cascini for his precious suggestions and fruitful discussions about functional based foresight.

The University Consortium on Quality and Innovation Engineering (Quinn) at the University of Pisa supported the publication of the final report in a book format.

Open Access This article is distributed under the terms of the Creative Commons Attribution 4.0 International License (http:// creativecommons.org/licenses/by/4.0/), which permits unrestricted use, distribution, and reproduction in any medium, provided you give appropriate credit to the original author(s) and the source, provide a link to the Creative Commons license, and indicate if changes were made.

\section{References}

1. Kannengiesser U, Gero JS (2012) A process framework of affordances in design. Des Issues 28(1):50-62

2. Fantoni G, Apreda R, Gabelloni D, Bonaccorsi A (2011) Do functions exist? Int Conf Eng Des, ICED11 2:304-313

3. Gabelloni D, Apreda R, Fantoni G (2011) On the link between features and functions. Int Conf Eng Des, ICED11 2:1-12
4. Hekkert MP, Suurs RAA, Negro SO, Kuhlmann S, Smits R (2007) Functions of innovation systems: a new approach for analysing technological change. Technol Forecast Soc Chang 74:413-432

5. Bonaccorsi, A, Fantoni, B (2007) Expanding the functional ontology in conceptual design. International Conference on Engineering Design, ICED'07. https://www.designsociety.org/downloadpublication/25716/expanding_the_functional_ontology_in conceptual_design. Accessed 4 October 2016

6. Bonaccorsi A, Apreda R, Fantoni G (2009) A theory of the constituent elements of functions. Int Conf Eng Des, ICED09 2: 179-190

7. Bergek A, Jacobsson S, Sandén B (2008) 'Legitimation' and 'development of positive externalities': two key processes in the formation phase of technological innovation systems. Technol Anal Strat Manag 20:575-592

8. Bergek A, Jacobsson S, Carlsson B, Lindmark S, Rickne A (2008) Analyzing the functional dynamics of technological innovation systems: a scheme of analysis. Res Policy 3:407-429

9. Hirtz JM, Stone RB, McAdams DA, Szykman S, Wood K (2002) A functional basis for engineering design: reconciling and evolving previous efforts. Res Eng Des 13:65-82. doi:10.1007/s00163-001-0008-3

10. Vermaas P (2007) The functional modelling account of stone and wood: some critical remark. International Conference on Engineering Design, ICED'07. http://pietervermaas. nl/PDF/Pieter_Vermaas_FUNENG_Stone_and_Wood.pdf. Accessed 4 October 2016

11. Ahmed S, Wallace KM (2003) Evaluating a functional basis. Proceedings of ASME 2003 Design Engineering Technical Conferences, DETC2003/DTM-48685. ASME

12. Bonaccorsi A (2011) A functional theory of technology and technological change. In: Antonelli C (ed) Handbook on the economic complexity of technological change. Edward Elgar, Cheltenham, pp 286-340

13. Altshuller GS (1984) Creativity as an exact science: the theory of the solution of inventive problems. Gordon and Breach Science Publishing, New York

14. Mann DL (2003) Better technology forecasting using systematic innovation methods. Technol Forecast Soc Chang 70(8):779-795

15. Fantoni G, Apreda R, Bonaccorsi A (2009) Functional vector space. Int Conf Eng Des, ICED09 2: 311-322

16. Kostoff RN, Boylan R, Simons GR (2004) Disruptive technology roadmaps. Technol Forecast Soc Chang 71(1-2):141-159

17. Kostoff RN, Schaller RR (2001) Science and technology roadmaps. IEEE Trans Eng Manag 48:132-143

18. Narin F (1994) Patent bibliometrics. Scientometrics 30:147-155

19. Narin F, Olivastro D (1988) Technology indicators based on patents and patent citations. In: Van Raan AFJ (ed) Handbook of quantitative studies of science and technology. Elsevier Publishers, North Holland

20. Meyer M (2001) Patent citation analysis in a novel field of technology: an exploration of nano-science and nano-technology. Scientometrics 51:163-183

21. Meyer M (2000) Patent citations in a novel field of technology. What can they tell about interactions between emerging communities of science and technology? Scientometrics 48:151-178

22. Kuusi O, Meyer M (2007) Anticipating technological breakthroughs: using bibliographic coupling to explore the nanotubes paradigm. Scientometrics 70:759-777

23. Verspagen B (2007) Mapping technological trajectories as patent citation networks: a study on the history of fuel cell research. Adv Complex Syst 10:93-115

24. Yoon B, Park Y (2004) A text-mining-based patent network: analytical tool for high technology trend. J High Technol Manag Res $15: 37-50$

25. Chang PL, Wu CC, Leu HJ (2010) Using patent analyses to monitor the technological trends in an emerging field of technology: a case of carbon nanotube field emission display. Scientometrics 82:5-19 
26. Hummon NP, Doreain P (1989) Connectivity in a citation network: the development of DNA theory. Soc Networks 11:39-63

27. Oudshoorn N (2011) Who cares? Telecare technologies and the transformation of healthcare. Palgrave MacMillan, London

28. Budde B, Alkemade F, Weber KM (2012) Expectations as a key to understanding actor strategies in the field of fuel cell and hydrogen vehicles. Technol Forecast Soc Chang 79-540(6-7):1072-1083

29. Apreda R, Bonaccorsi A, Fantoni G, Gabelloni D (2011) Functions and failures. How to manage technological promises for societal challenges. Technol Anal Strat Manag 26:369-384

30. Bonaccorsi A, Fantoni G, Apreda R (2013) Progetto di Foresight Tecnologico: Metodi e tecniche non convenzionali per immaginare il futuro della tecnologia. University Press, Pisa

31. Eames M, Stirling A, Burgess J, Davies G, Williamson S, Mayer S, Stanley K (2003) Deliberative mapping: integrating citizens and specialists appraisals in a transparent and inclusive participatory process. Paper presented at FRONTIERS 2: european applications in ecological economics, www.euroecolecon.org/frontiers/contributions//F2 papers/FD8.pdf. Accessed 4 October 2016

32. Fantoni G, Taviani C, Santoro R (2006) Design by functional synonyms and antonyms: a structured creative technique based on functional analysis. Proc Inst Mech Eng B J Eng Manuf 221(4):673-683

33. Fantoni G, Gabelloni D, Tilli J (2013) Concept design of new grippers using abstraction and analogy. Part B: J Eng Manuf 227(10): $1521-1532$
34. Dell'Orletta F, Venturi G, Cimino A, Montemagni S (2014) T2K²: a system for automatically extracting and organizing knowledge from texts. Proceedings of 9 th edition of international conference on language resources and evaluation, 2014. pp 2062-2070

35. Ferrari A, Oronzo Spagnolo G, Dell'Orletta F (2013) Mining commonalities and variabilities from natural language documents. Proceedings of the 17th international software product line conference, SPLC-2013. pp 116-120

36. Fantoni G, Apreda R, Dell'Orletta F, Monge M (2013) Automatic extraction of function-behaviour-state information from patents. J Adv Eng Inform 27(3):317-334

37. Ferrari A, Dell'Orletta F, Oronzo Spagnolo G, Gnesi S (2014) Measuring and improving the completeness of natural language requirements, Proceedings of the 20th international working conference on requirements engineering: foundation for software quality, REFSQ 2014. 8396: 23-38

38. Bonin F, Dell'Orletta F, Montemagni S, Venturi G (2010) A contrastive approach to multi-word extraction from domain-specific corpora. Proceedings of the 7th international conference on language resources and evaluation, LREC 2010. pp 3222-3229

39. Frantzi K, Ananiadou S (1999) The C-value/NC Value domain independent method for multi-word term extraction. J Nat Lang Process 6(3):145-179

40. Kononiuk A, Sacio-Szymańska A (2015) Assessing the maturity level of foresight in Polish companies - a regional perspective. Eur J Futur Res 3:23. doi:10.1007/s40309-015-0082-9 